\title{
Reconstruction of the medial patellofemoral ligament for painful patellar subluxation in distal torsional malalignment: a case report
}

\author{
Schoettle, P B ; Werner, C M L ; Romero, J
}

\begin{abstract}
Complex two-level rotational malalignment of the lower extremity can cause maltracking of the patella with anterior knee pain. Double derotation osteotomy would correct the underlying pathology. However, it carries a high risk of complications such as nerve and vessel damage. We report a case of rotational malalignment in the femur and the tibia associated with trochlear dysplasia, which causes painful patellar instability. The patient was successfully treated with reconstruction of the medial patellofemoral ligament and lateral release. Although the malrotation was not addressed, the position of the patella was corrected, and no dislocation occurred during a follow-up of 10months
\end{abstract}

DOI: https://doi.org/10.1007/s00402-005-0822-6

Posted at the Zurich Open Repository and Archive, University of Zurich ZORA URL: https://doi.org/10.5167/uzh-156647

Journal Article

Published Version

Originally published at:

Schoettle, P B; Werner, C M L; Romero, J (2005). Reconstruction of the medial patellofemoral ligament for painful patellar subluxation in distal torsional malalignment: a case report. Archives of Orthopaedic and Trauma Surgery, 125(9):644-648.

DOI: https://doi.org/10.1007/s00402-005-0822-6 


\section{Reconstruction of the medial patellofemoral ligament for painful patellar subluxation in distal torsional malalignment: a case report}

Received: 24 June 2004 / Published online: 2 July 2005

(C) Springer-Verlag 2005

\begin{abstract}
Complex two-level rotational malalignment of the lower extremity can cause maltracking of the patella with anterior knee pain. Double derotation osteotomy would correct the underlying pathology. However, it carries a high risk of complications such as nerve and vessel damage. We report a case of rotational malalignment in the femur and the tibia associated with trochlear dysplasia, which causes painful patellar instability. The patient was successfully treated with reconstruction of the medial patellofemoral ligament and lateral release. Although the malrotation was not addressed, the position of the patella was corrected, and no dislocation occurred during a follow-up of 10 months.
\end{abstract}

Keywords Distal torsional alignment $\cdot$ Medial patellofemoral ligament · Patellar instability ·

Reconstruction $\cdot$ Knee

\section{Introduction}

Torsional malalignment syndrome of the lower extremity is the result of excessive medial femoral and lateral tibial torsion and can cause severe knee pain [26]. Most torsional problems of childhood (excessive femoral anteversion, internal or external tibial rotation) resolve with age [13]. Only a small percentage of older children and adults suffer from functional or cosmetic problems, which require surgical treatment [29]. The procedures consist of corrective osteotomies, in which the distal part of the tibia is internally rotated or the distal part of the

P. B. Schoettle · C. M. L. Werner · J. Romero

Department of Orthopaedics, University of Zurich,

Balgrist, Switzerland

J. Romero $(\bowtie)$

Uniklinik Balgrist, Forchstr. 340, 8008 Zürich, Switzerland

E-mail: jose.romero@balgrist.ch

Tel.: +41-1-3861111

Fax: + 41-1-3861609 femur is externally rotated. In severe cases, derotation osteotomies on both levels (tibial and femur) may be necessary [8]. Such two-level osteotomies have several disadvantages such as atrophy of muscular soft tissue, and the risk of non-union or nerve injuries [31].

We report on the surgical treatment of a patient with combined femoral and tibial malrotation associated with trochlear dysplasia grade III who suffered from anterior knee pain and recurrent patellar dislocation.

\section{Case report}

A 15-year-old girl presented with an internally rotated distal femur and an externally rotated proximal tibia of her right leg. Severe anterior knee pain due to a complete patellar dislocation at full extension and permanent apprehension were her main complaints. First time dislocation occurred while stair walking at the age of 9 years without prior trauma. Ever since, she has suffered from recurrent patellar dislocation with spontaneous relocation.

Clinical examination revealed an inverted knee joint, but no in-toeing during gait. Her spontaneous foot position was in slight external rotation in supine position.

Patellofemoral mobility was increased compared with the contralateral side, and a positive apprehension was present on the index knee. The menisci and cruciate ligaments were without pathological findings. Additional tenderness of the medial patellofemoral complex was present. In full extension, the patella was completely dislocated (Fig. 1). In $30^{\circ}$ of flexion the patella glided on the lateral part of the lateral femoral condyle (Fig. 2). Straight lateral X-rays revealed a trochlear dysplasia grade III [7] (Fig. 3).

Femoral anteversion was measured on CT scan using the technique described by Lerat et al. [18]. Femoral antetorsion is the summation of the angle of the bicondylar line to a horizontal line (Fig. 4A) and the angle of a line between the geometrical femoral head centre and the mid-basis of the femoral neck to the same 


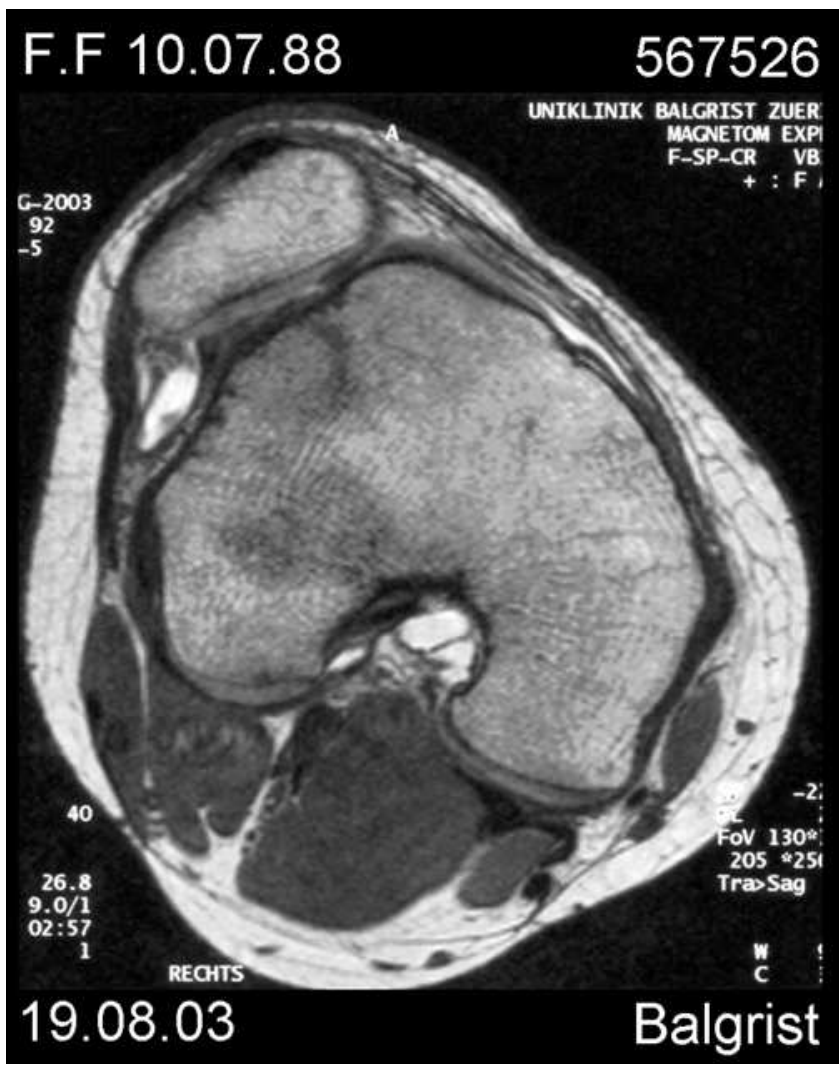

Fig. 1 Preoperative MRI scan showing arthritic deformations of the trochlea and a subluxated patella

horizontal line (Fig. 4B). The calculated anteversion was $28.3^{\circ}$, while normal femoral anteversion is between $10^{\circ}$ to $14^{\circ}[1,16,17,33]$. Using the technique of Jend et al. [17], tibial rotation was $51^{\circ}$ (Fig. 5), while normal values

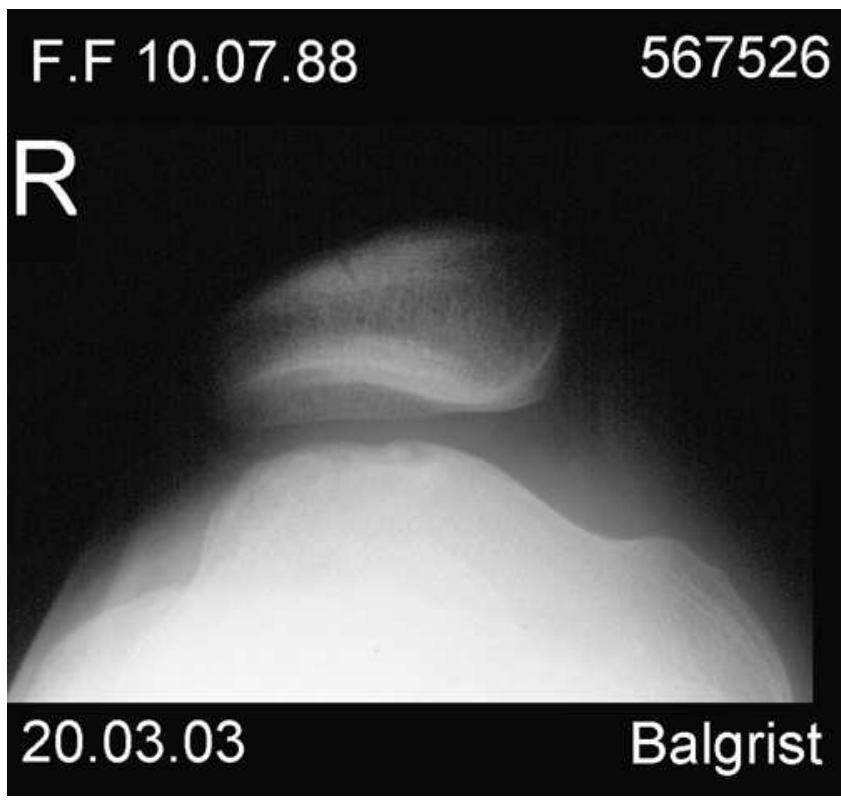

Fig. 2 Preoperative axial view

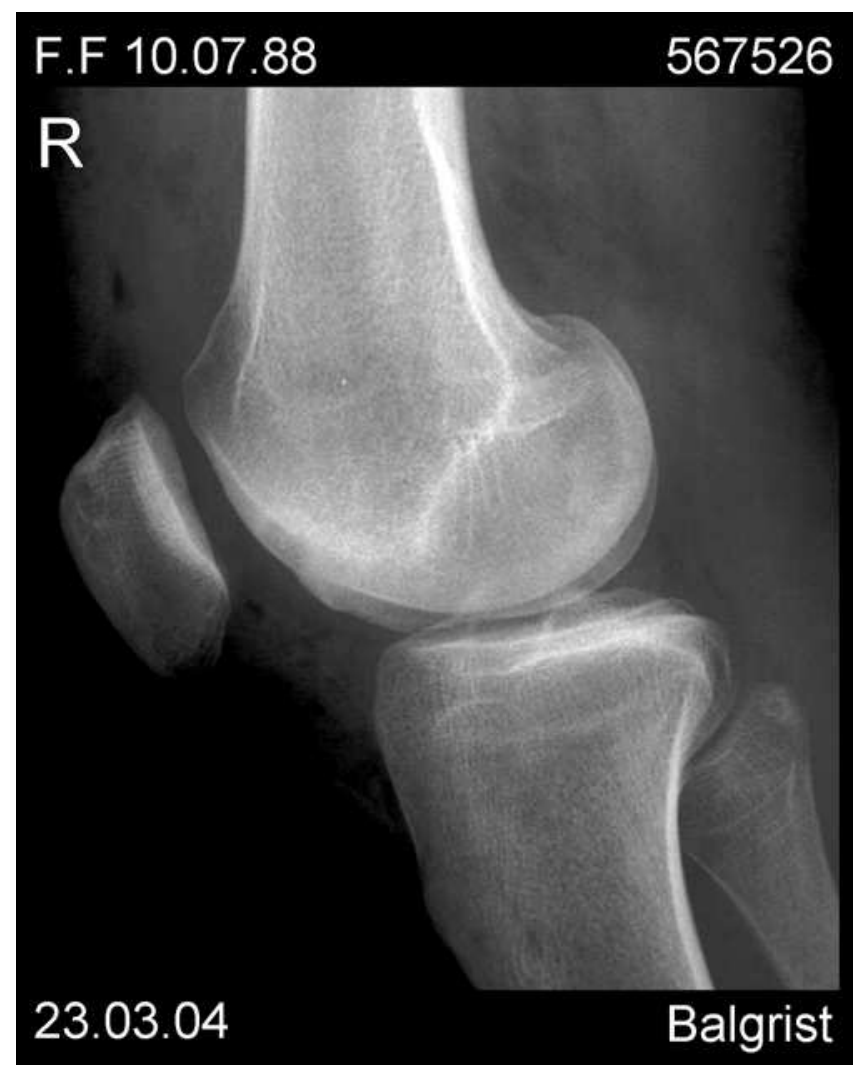

Fig. 3 Straight lateral view with a positive crossing sign grade III and a trochlear bump

are between $20^{\circ}$ and $40^{\circ}[1,12,17,27,28,30]$. That resulted in a total external rotation of $7^{\circ}$, estimating that mean femoral anteversion is $12^{\circ}$ and mean tibial external rotation is $30^{\circ}$.

Tibial tuberosity-trochlear groove distance (TTTG) was $24 \mathrm{~mm}$, while normal values are below $15 \mathrm{~mm} \mathrm{[2,7,}$ 14] (Fig. 6). MRI examination depicted concomitant cartilaginous lesions with arthritic deformation of the trochlea and a lateral trochlear inclination of $2^{\circ}$, which is an additional sign of trochlear dysplasia [6] (Fig. 1).

Since overall rotation of the lower extremity was within acceptable limits, and patellar dislocation was the chief complaint, only a soft-tissue procedure was chosen to correct patellar maltracking. The technique utilises an ipsilateral semitendinosus graft for the reconstruction of the medial patellofemoral ligament (MPFL). The proximal one-third aspect of the patella is exposed, and a bony groove is made. Two suture anchors carrying no. 3 non-absorbable braided suture are placed into that groove. A guidewire is placed into the adductor tubercle across the epicondyles from an additional short incision. A hole of $6 \mathrm{~mm}$ diameter and $2.5 \mathrm{~cm}$ depth was drilled over the guidewire. Then, the semitendinosus tendon is harvested through a short oblique incision of $2-3 \mathrm{~cm}$. The graft is then folded in half, and the centre portion is sutured to the anchors at the patella. The two free endings of the semitendinosus graft are pulled into the 
Fig. 4 A Distal femoral scan with an angle of $23.9^{\circ}$ between the bicondylar line and a horizontal line. B Femoral neck scan with an angle of $4.4^{\circ}$ between femoral neck and a horizontal line
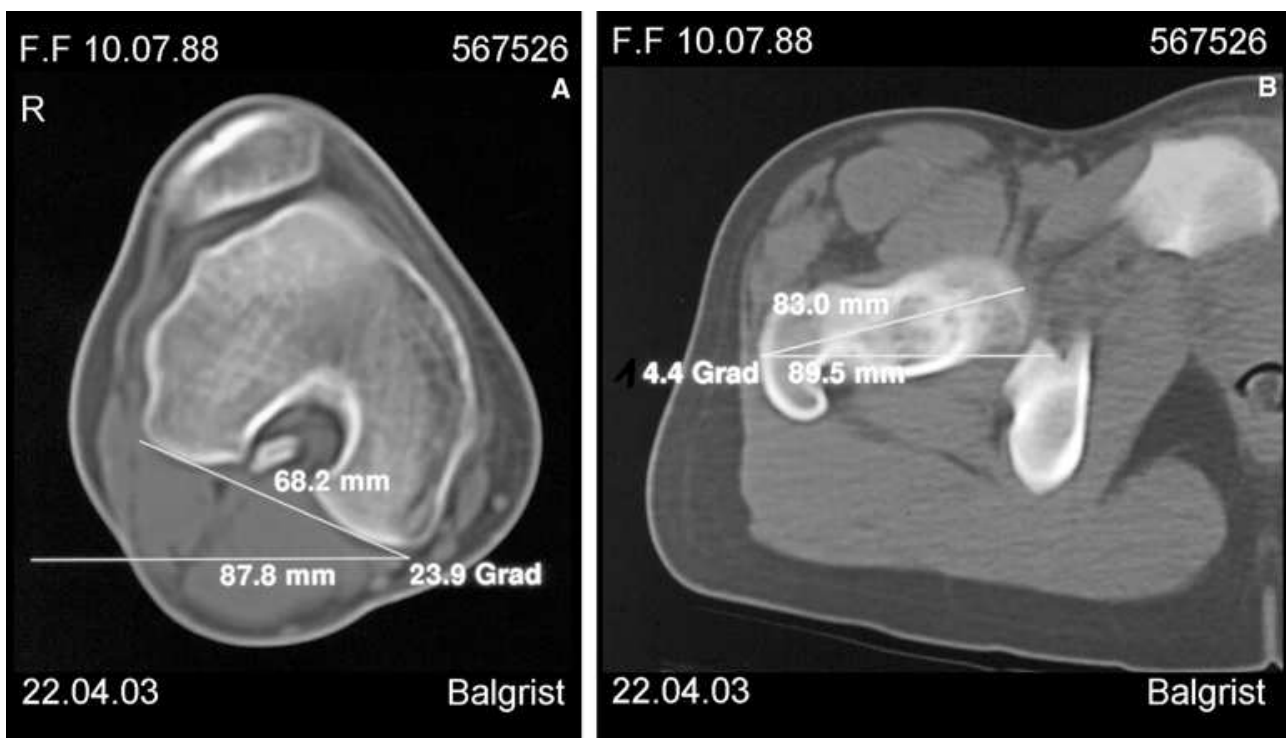

drill hole at the adductor tubercle by removing the guidewire through the medial epicondyle. Tensioning of the graft is performed according to a realigned patellar tracking throughout the knee's range of motion. An absorbable soft-tissue interference screw is then inserted into the drill hole at the adductor tubercle $[10,19,24]$.

Postoperatively, the patella had a correct alignment when the patient left hospital after 5 days. At the 10month follow-up visit she reported on substantially decreased pain and absence of patellar apprehension.

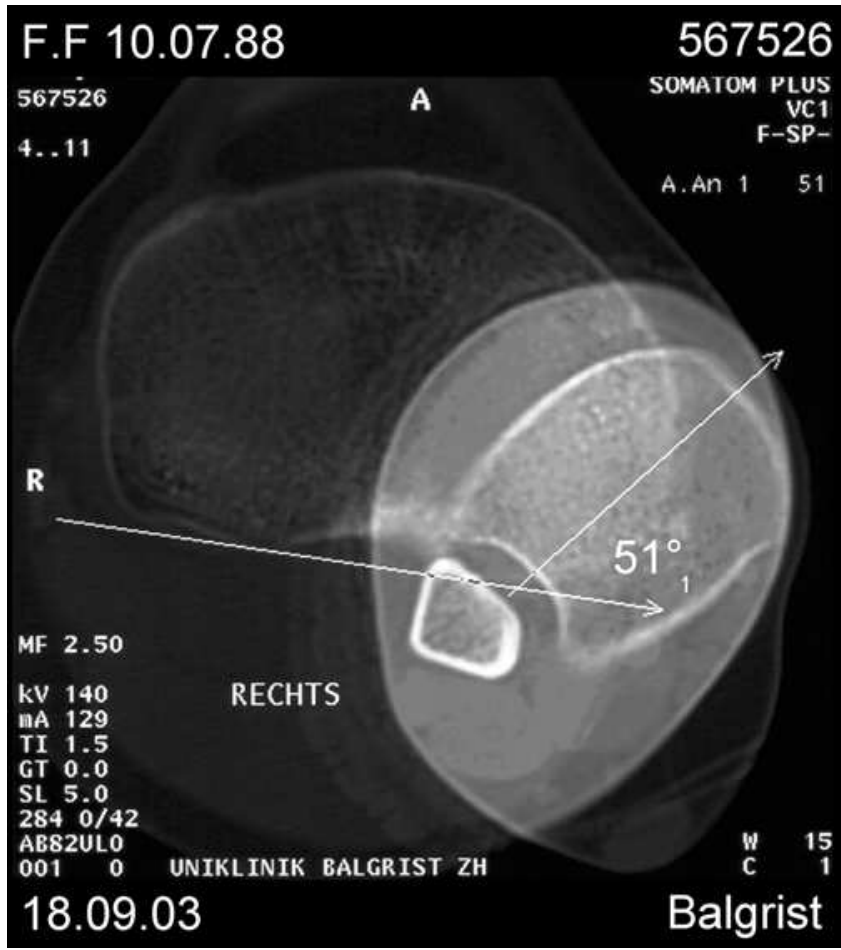

Fig. 5 Rotation scans of the tibial head and the ankle with a tibial torsion angle between the posterior tibial line and the malleolar line of $51^{\circ}$
Crepitus was absent during knee motion, and the patella was completely aligned throughout the full range of motion. The axial X-ray view at $30^{\circ}$ of knee flexion revealed complete patellofemoral realignment (Fig. 7). She was allowed to return to sports activity such as jogging and swimming as well as contact sports like basketball after the 10-month visit.

\section{F.F 10.07 .88} 567526

16. .27
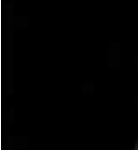

\section{6} SOMAT
A

Di 1 An 1

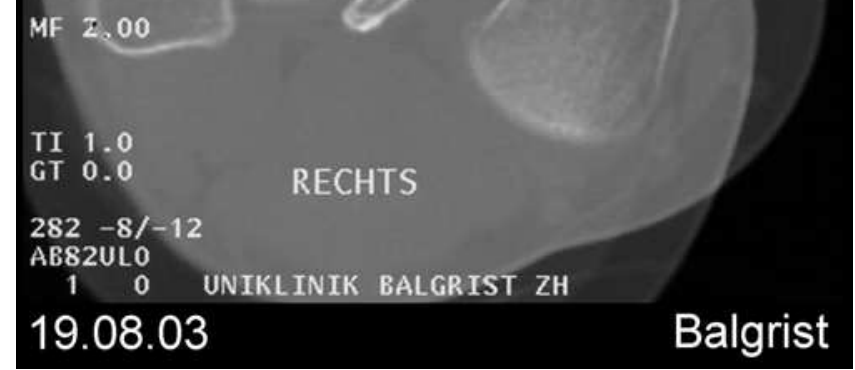

Fig. 6 Measurement of the TTTG (tibial tuberosity-trochlear groove distance) 


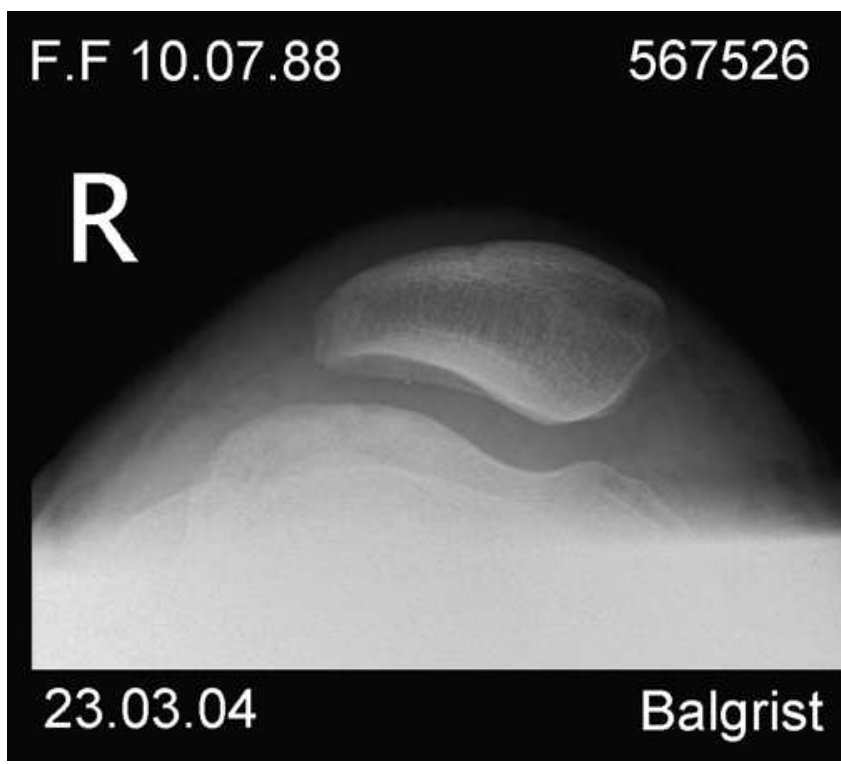

Fig. 7 Postoperative axial view with a lateral patellar tilt of $14^{\circ}$

\section{Discussion}

Distal torsional malalignment syndrome occurs as a result of excessive internal rotation of the femur and external rotation of the tibia, resulting in an abnormal joint motion [26]. The axis of the knee motion is medially rotated, and the patella assumes what appears to be a curvilinear pathway from flexion to extension: a $\mathbf{J}$ sign. This rotational malalignment may lead to patellofemoral maltracking and patellar dislocation.

In patients with patellofemoral symptoms, femoral anteversion was found to be increased [21, 32, 33]. Another study showed an increase in both femoral antetorsion and lateral tibial torsion [1], leading to recurrent patellar instability. Correction of two-level rotational malalignment would require osteotomies at femoral and tibial sites. Such complex osteotomies have a high complication rate (up to $15 \%$ ). Peroneal nerve injuries, compartment syndrome, non-union, delayed union, malunion and infection have been reported after rotational osteotomy $[3,25]$. Such a complex surgical procedure is therefore seldom justified in the face of the patient's moderate complaints, particularly if the patients have been operated on for only in-toeing and other cosmetic reasons. Delgado et al. reported on 8 patients treated without any complications [8]. None of the cases with two-level osteotomies described until now had a concomitant trochlear dysplasia. Since conservative treatment has been found to have limited success in alleviating anterior knee pain [8], double-level osteotomy might reduce pain and improve patellar maltracking [28]. Two-level osteotomy, however, would not influence the patellar instability caused by trochlear dysplasia and would not address the torn medial patellofemoral structures. An additional trochleaplasty was contrain- dicated in this specific patient due to overt degenerative changes of the trochlea $[4,23]$. Hence, the treatment aim of this patient was to correct painful lateral patellofemoral maltracking and to eliminate discomfort due to persistent apprehension, without exposing the patient to potential complications encountered with osteotomies. The MPFL is known to be ruptured or at least insufficient after first time patellar dislocation [22]. Cadaver studies have proven the importance of the medial soft tissue in resisting lateral patellar motion. The MPFL as a primary stabilizer of the patella contributes $40-80 \%$ of the total medial restraining force $[5,9,15,19]$. Reconstruction of the MPFL has been advocated by several authors for patellofemoral instability as it realigns the patella more effectively than proximal or distal realignment procedures. A success rate of more than $90 \%$ has been reported in the literature with a very low complication rate [10, 11, 24]. Furthermore, a retrospective clinical study found reconstruction of the MPFL to be successful also for patellar instability with concomitant low-grade trochlear dysplasia (grades I and II) [24]. Isolated lateral release is not recommended as an intervention since it has been proved that lateral release alone can lead to medial patellofemoral instability and increased medial patellofemoral pressure [20].

To our knowledge, no case of surgical treatment of distal torsional alignment and patellofemoral instability with concomitant trochlear dysplasia grade III has been reported so far, either with a two-level osteotomy or with a soft-tissue reconstruction. MPFL reconstruction reduced the subjective discomfort of patellar instability and painful lateral maltracking in this patient, although the primary pathologies (torsional malalignment and trochlear dysplasia) were not addressed.

There are limitations to the conclusions drawn in this case report, in that the follow-up only lasted 10 months. We are aware that a follow-up of less than 2 years is too short to report on the definite outcome of this patient. However, this excellent result at early follow-up of 10 months is promising and unlikely to change over time. If revision should be necessary in the future in case of redislocation, we would perform the two-level osteotomy with additional trochleaplasty, thus eliminating all factors contributing to the patellar instability.

\section{References}

1. Airanow S, Zippel H (1990) Untersuchung zur femoro-tibialen Torsion bei Patellainstabilitaten. Ein Beitrag zur Pathogenese rezidivierender und habitueller Patellaluxationen. Beitr Orthop Traumatol 37:311-316

2. Beaconsfield T, Pintore E, Maffulli N, Petri GJ (1994) Radiological measurements in patellofemoral disorders. A review. Clin Orthop 308:18-28

3. Bennett JT, Bunnell WP, MacEwen GD (1985) Rotational osteotomy of the distal tibia and fibula. J Pediatr Orthop 5:294-298

4. Bereiter H, Gautier E (1994) Die Trochleaplastik als Chirurgische Therapie der Rezidivierenden Patellaluxation bei Trochleadysplasie. Arthroskopie 7:281-286 
5. Burks RT, Desio SM, Bachus KN, Tyson L, Springer K (1998) Biomechanical evaluation of lateral patellar dislocations. Am J Knee Surg 11:24-31

6. Carrillon Y, Abidi H, Dejour D, Fantino O, Moyen B, TranMinh VA (2000) Patellar instability: assessment on MR images by measuring the lateral trochlear inclination-initial experience. Radiology 216:582-585

7. Dejour H, Walch G, Nove-Josserand L, Guier C (1994) Factors of patellar instability: an anatomic radiographic study. Knee Surg Sports Traumatol Arthrosc 2:19-26

8. Delgado ED, Schoenecker PL, Rich MM, Capelli AM (1996) Treatment of severe torsional malalignment syndrome. J Pediatr Orthop 16:484-488

9. Desio SM, Burks RT, Bachus KN (1998) Soft tissue restraints to lateral patellar translation in the human knee. Am J Sports Med 26:59-65

10. Drez D Jr, Edwards TB, Williams CS (2001) Results of medial patellofemoral ligament reconstruction in the treatment of patellar dislocation. Arthroscopy 17:298-306

11. Ellera Gomes JL, Stigler Marczyk LR, Cesar de Cesar P, Jungblut CF (2004) Medial patellofemoral ligament reconstruction with semitendinosus autograft for chronic patellar instability: a follow-up study. Arthroscopy 20:147-151

12. Fabry G (1997) Normal and abnormal torsional development of the lower extremities. Acta Orthop Belg 63:229-232

13. Fabry G (1977) Torsion of the femur. Acta Orthop Belg 43:454-459

14. Goutallier D, Bernageau J, Lecudonnec B (1978) Mesure de l'écart tubérosité antérieure-gorge de la trochlée. Rev Chir Orthop Reparatrice Appar Mot 64:423-428

15. Hautamaa PV, Fithian DC, Kaufman KR, Daniel DM, Pohlmeyer AM (1998) Medial soft tissue restraints in lateral patellar instability and repair. Clin Orthop 349:174-182

16. Janssen G (1984) Der Innenrotationsfehler des Kniegelenkes als Ursache der Chondromalacia patellae. Swiss Med 6:53-55

17. Jend HH, Heller M, Schontag H, Schoettle H (1980) Eine computertomographische Methode zur Bestimmung der Tibiatorsion. Rofo 133:22-25

18. Lerat JL, Moyen B, Grammont P (1985) Die klinische Untersuchung der unteren Extremitat in den drei Ebenen. Beitrag der Tomodensitometrie zur Analyse in der horizontalen Ebene. Orthopade 14:215-219
19. Nomura E, Horiuchi Y, Kihara M (2000) Medial patellofemoral ligament restraint in lateral patellar translation and reconstruction. Knee 7:121-127

20. Nonweiler DE, DeLee JC (1994) The diagnosis and treatment of medial subluxation of the patella after lateral retinacular release. Am J Sports Med 22:680-686

21. Reikeras O (1992) Patellofemoral characteristics in patients with increased femoral anteversion. Skeletal Radiol 21:311-313

22. Sallay PI, Poggi J, Speer KP, Garrett WE (1996) Acute dislocation of the patella. A correlative pathoanatomic study. Am J Sports Med 24:52-60

23. Schottle PB, Fucentese SF, Pfirrmann CM, Bereiter H, Romero J (2004) Trochleaplasty for patellofemoral instability due to trochlear dysplasia - a minimum 2-year clinical and radiological follow-up of 19 knees. Acta Orthop Scand (in press)

24. Schottle PB, Fucentese SF, Romero J (2004) The reconstruction of the medial patellofemoral ligament for the unstable patella - a clinical and radiological outcome study. Knee Surg Sports Traumatol Arthrosc (accepted for publication)

25. Schrock RD Jr (1969) Peroneal nerve palsy following derotation osteotomies for tibial torsion. Clin Orthop 62:172-177

26. Staheli LT (1980) Medial femoral torsion. Orthop Clin North Am 11:39-50

27. Staheli LT (1987) Rotational problems of the lower extremities. Orthop Clin North Am 18:503-512

28. Staheli LT (1977) Torsional deformity. Pediatr Clin North Am 24:799-811

29. Staheli LT (1989) Torsion - treatment indications. Clin Orthop 247:61-66

30. Staheli LT, Engel GM (1972) Tibial torsion: a method of assessment and a survey of normal children. Clin Orthop 86:183-186

31. Svenningsen S, Apalset K, Terjesen T, Anda S (1989) Osteotomy for femoral anteversion. Complications in 95 children. Acta Orthop Scand 60:401-405

32. Takai S, Sakakida K, Yamashita F, Suzu F, Izuta F (1985) Rotational alignment of the lower limb in osteoarthritis of the knee. Int Orthop 9:209-215

33. Yamashita F, Sakakida K (1988) The rotational alignment of the lower limbs in recurrent dislocation of the patella. Nippon Geka Hokan 57:215-220 